Vol. 21, n 1 | 2017

Varia

Le vagabondage, ou la police des existences irrégulières et incertaines : sens et usages d'un délit (France, 1815-1850)

\title{
Pierre Gaume
}

\section{OpenEdition}

\section{Journals}

Electronic version

URL: http://journals.openedition.org/chs/1718

DOI: $10.4000 /$ chs. 1718

ISSN: 1663-4837

\section{Publisher}

Librairie Droz

\section{Printed version}

Date of publication: 1 January 2017

ISSN: 1422-0857

\section{Electronic reference}

Pierre Gaume, «Le vagabondage, ou la police des existences irrégulières et incertaines : sens et usages d'un délit (France, 1815-1850) », Crime, Histoire \& Sociétés / Crime, History \& Societies [Online], Vol. 21, $n^{\circ} 1$ | 2017, Online since 01 January 2019, connection on 10 December 2020. URL : http:// journals.openedition.org/chs/1718; DOI : https://doi.org/10.4000/chs.1718

This text was automatically generated on 10 December 2020.

(C) Droz 


\title{
Le vagabondage, ou la police des existences irrégulières et incertaines : sens et usages d'un délit (France, 1815-1850)
}

\author{
Pierre Gaume
}

En février 1821, inquiet des « vols multipliés » qui se commettent dans le département, le préfet du Rhône s'adresse ainsi aux maires :

«Messieurs,

[...] J'ai donné à la gendarmerie royale les ordres et les instructions nécessaires pour qu'elle ait à redoubler de zèle et d'activité dans la recherche des malfaiteurs ; mais [...] ces efforts seraient insuffisants, si elle n'était efficacement secondée dans ses recherches et dans son action par MM. les maires, leurs adjoints et les gardes champêtres [...].

Je fixerai particulièrement votre attention sur les mendiants et vagabonds voyageant sans titre et qui, dans cet état, n'offrent aucune garantie à la société. Ces individus se répandent presque sans obstacle dans les campagnes; sous le prétexte de mendier, quelques-uns observent les maisons isolées, et saisissent les occasions pour y commettre des vols. D'autres sont des individus frappés de condamnations ou de mandats de justice à l'exécution desquels ils se sont soustraits. C'est à cette classe errante que l'on doit attribuer la plupart des délits qui se commettent dans les campagnes.

Or, il est évident que si les lois et règlements sur les passeports étaient exécutés avec soin, si l'autorité locale faisait arrêter et remettre entre les mains de la gendarmerie tous les individus qui, étant étrangers au département, s'y présentent sans passeport et dans un véritable état de vagabondage il est évident, dis-je, que les communes seraient préservées des délits auxquels les expose le défaut de surveillance envers ces vagabonds $»^{1}$.

Confronté à un climat d'inquiétude sécuritaire, source de « justes alarmes », le préfet s'en remettait à l'arme policière qui lui paraissait la plus adaptée : à travers la surveillance des étrangers et le contrôle des mobilités ${ }^{2}$, grâce à l'arrestation des individus suspects, en état de vagabondage, les auteurs de vols ne manqueraient pas d'être arrêtés ou, du moins, 
forcés de s'éloigner. De telles instructions devaient se répéter en bien d'autres occasions, constituant une réponse, ici à l'apparition de bandes inquiétant les populations, là au mouvement d'ouvriers sans emploi vers les grandes agglomérations, etc. En agissant ainsi, ce magistrat s'inscrivait en effet dans une tradition historique, forgée au cours de l'époque moderne, qui tendait à faire du vagabondage un instrument essentiel du maintien de l'ordre, adossé à une police des circulations qui connut, elle aussi, d'importants développements. Après les travaux de J.-P. Gutton, B. Geremek ou R. Castel, plusieurs études majeures sont venues éclairer ce processus ${ }^{3}$. À travers les lois et ordonnances incriminant le vagabondage, il était possible de lire l'affirmation d'une exigence croissante de préservation de l'ordre public, fixant l'attention de la police sur les classes populaires et leurs mobilités, perçues comme sources de désordre potentiel. Cette "police des pauvres", ainsi que la désignent les historiens anglo-saxons, fut une part importante de l'activité ordinaire des agents, en ville comme à la campagne ${ }^{4}$. Dans cette perspective, le délit de vagabondage eut un rôle considérable : le caractère relativement indéterminé, ouvert, de l'incrimination rendait possible l'interpellation de nombreux individus menant une même existence irrégulière ou incertaine, à la lisière de l'ordre légal, suivant des contours qui connurent des variations importantes - juristes et historiens ont pu parler de délit « attrape-tout » à ce sujet ${ }^{5}$. L'adoption d'ordonnances ou de lois répressives, à l'image des Vagrancy laws anglaises, tout comme l'inscription du délit dans le Code pénal, en 1810, consacraient donc l'avènement d'une police "préventive " qui, au nom de la dangerosité présumée de certaines populations, des préjugés entourant leurs modes d'existence, autorisait leur arrestation et leur condamnation, sur la base de cette seule présomption ${ }^{6}$. Malgré les réticences de certains magistrats, gênés d'une telle entorse au droit, ce processus conduisit souvent l'appareil judiciaire à imaginer des procédures rapides, hâtives, où les cas étaient traités de façon sérielle - consacrant le nouveau rôle de la police dans la chaîne pénale ${ }^{7}$. La loi de 1863 sur le flagrant délit en fut un des exemples les plus marquants ${ }^{8}$. Ce rôle historique du vagabondage connut sans doute son apogée entre la fin du XIX ${ }^{\mathrm{e}}$ siècle et le début du XX ${ }^{\mathrm{e}}$ siècle, période marquée par une envolée des arrestations et des condamnations, ainsi que par une inflation discursive sur ce thème ${ }^{9}$. Tandis que s'affirmait le souci d'une nouvelle prise en charge, marquant les débuts de l'État social, les crises et les bouleversements économiques conduisaient à interroger les partages à l'œuvre. Si l'idée d'une assistance aux vieillards et aux invalides parvint à s'imposer, la question des valides restait problématique: parmi la masse d'individus sans travail, il était nécessaire de distinguer entre les chômeurs involontaires, victimes malheureuses, et les paresseux volontaires, mendiants et vagabonds professionnels appelant une juste répression ${ }^{10}$. Du fait de cette double inflation, statistique et discursive, les historiens ont eu tendance à se focaliser sur cette période, des années 1870 aux années 1910, voire sur l'entre-deux-guerres, délaissant parfois le premier XIX ${ }^{\mathrm{e}}$ siècle.

3 Aussi cet article se propose-t-il de revenir sur le sens et les usages pratiques du délit de vagabondage en France, entre 1815 et 1850, au lendemain de sa codification définitive, en 1810. Il s'agira d'abord de montrer combien cette codification a pu contribuer à faire du vagabondage un instrument important du maintien de l'ordre : relevant d'une logique de police préventive, le délit rendait possible l'incrimination d'une large gamme d'individus jugés suspects, au nom de leur incapacité commune à répondre d'un ancrage positif dans l'ordre légal (défaut de ressources et de domicile avéré, c'est-à-dire d'attaches communautaires ou professionnelles). Afin, toutefois, de mieux saisir le type exact de 
police que le vagabondage permit de mettre en œuvre, les populations qu'il se donnait pour cible, ses liens avec la réglementation des circulations devront être interrogés. Audelà des seuls papiers, dont l'importance reste essentielle, il fut aussi le contrepoint d'un important effort réglementaire, visant à encadrer et à limiter les mobilités des populations pauvres. Aux tentatives d'organiser des secours localisés, à destination des indigents domiciliés, répondait l'arrestation des individus qui, dénués de ressources, persistaient à circuler. Aussi les "étrangers ", inconnus du lieu, furent-ils l'une des principales cibles de l'action policière - mais non la seule. Après avoir délimité l'horizon d'application du délit, nous tenterons d'en éclairer la mise en œuvre concrète, pratique, au service de cette police des existences irrégulières, incertaines, qui fut une part importante de l'activité des forces de l'ordre. En effet, au-delà de l'arrestation, le délit ouvre la voie à une gestion complexe, à la croisée des appareils policier, administratif et judiciaire. Loin de conduire systématiquement à l'inculpation, l'interpellation débouche fréquemment sur des formes d'accommodement, des mesures d'expulsion ou de rapatriement, etc. Confrontés à des réalités individuelles très diverses, avec, à leur disposition, des moyens d'intervention et d'investigation limités, les forces de l'ordre, les autorités administratives (maires, préfets) et les magistrats doivent opérer un travail quotidien d'orientation et de triage. Cela ne va pas sans provoquer de tensions et de conflits entre ces différents acteurs, qui peinent parfois à s'accorder sur l'acception du délit. La répression du vagabondage obéit également à des temporalités et à une géographie dont nous essaierons de dégager certains traits, pistes de réflexion appelant des précisions ultérieures. Elle doit, enfin, composer avec des obstacles et des limites importants, dans le cadre d'une étatisation limitée, où les considérations et les réalités locales continuent souvent de prévaloir sur l'application uniforme du droit et de la loi. Terminons en évoquant les sources utilisées. Afin de saisir la signification sociohistorique du délit, nous avons essayé d'articuler différents types d'archives, à différentes échelles. Fruit de recherches menées dans le Rhône, notre corpus était d'abord composé de plus de 200 dossiers individuels, en partie issus des fonds de la préfecture, en partie du tribunal de Villefranche-sur-Saône ${ }^{11}$. Parfois sommaires, souvent incomplets, ils permettaient cependant d'appréhender les modalités de gestion des individus après leur interpellation, au-delà des seules réalités statistiques consignées dans les rôles des greffes et les registres des prisons. Afin d'élargir le propos, cette documentation a été confrontée à de nombreux courriers officiels et administratifs : le dépouillement des arrêtés et circulaires du préfet du Rhône fut ainsi complété par des textes issus d'autres départements, recueillis lors d'un travail précédent ${ }^{12}$. Il le fut aussi par la consultation des archives du ministère de l'Intérieur, et par la très riche correspondance du ministère de la Justice, conservée dans la série $\mathrm{BB}^{18}$ des Archives nationales (CARAN). Dans ce même souci d'élargir notre propos, la lecture de la Gazette des tribunaux s'est avérée précieuse, éclairant la pratique judiciaire. Enfin, n'étant pas familier du droit, nous avons dû consulter les auteurs de la doctrine, afin d'apprécier au mieux le sens de la législation. 


\section{Le vagabondage, instrument juridique d'une police préventive?}

\section{"Incrimination préventive ", « délit de suspicion »13 : le vagabondage dans le Code pénal de 1810}

4 La spécificité du délit de vagabondage fut, en effet, très tôt relevée par les juristes et les historiens du droit ${ }^{14}$ : suivant une phraséologie héritée de l'Ancien Régime, l'article 270 n'incrimine aucun fait positif mais une situation, un état social défini de façon purement négative, ce que J.-F. Chassaing appelait une «infraction de comportement $»^{15}$. Plus surprenant, le délit permet d'inverser la charge de la preuve, suivant une logique accusatoire : c'est au prévenu, suspect a priori, qu'il revient de prouver la réalité de son domicile et de ses moyens d'existence, que ce soit en produisant des papiers ou des attestations, en faisant appel à des individus capables de répondre de lui, ou par des déclarations suffisamment précises pour être vérifiées. Le vagabondage dessine donc les contours d'un délit de suspicion, qui vient sanctionner l'incapacité à produire des garanties d'un état - en fait d'un ancrage positif dans la société et l'ordre légal. C'est bien à ce titre que M. Raymond est inculpé en 1826 :

«Cet individu ne donnant aucune preuve à l'appui de ses assertions, et refusant d'ailleurs de fournir les renseignements propres à vérifier sa position, il y a lieu de le considérer comme vagabond et sans aveu $»^{16}$.

Six ans plus tard, P. Trouillé est condamné pour cette même raison, du fait du mystère entourant son existence ${ }^{17}$. Particulièrement suspect dans un contexte de généralisation des papiers, cet état d'incertitude et d'indétermination sociale vaut donc présomption de culpabilité. Le délit est, par ailleurs, fortement marqué par la logique de l'aveu, héritée des périodes précédentes: l'incapacité à se faire réclamer est un puissant motif de suspicion, qui peut mener à l'inculpation. F. Massot,

6 J. Vercker ou M. Auclair sont tous considérés comme vagabonds pour n'avoir pu se recommander de personne ${ }^{18}$. À l'inverse, l'intervention d'un "réclamant " entraine l'annulation des poursuites, fait peu ordinaire dans la procédure pénale ${ }^{19}$.

7 Les sanctions associées au délit confirment son statut particulier. Si l'article 271 prévoit un emprisonnement de trois à six mois, rapidement modéré par l'application des circonstances atténuantes ${ }^{20}$, la peine peut être portée à cinq ans dans certains cas aggravés, renforçant la suspicion d'intentions coupables (travestissement, port d'armes ou d'objets pouvant servir à des vols) ${ }^{21}$. Plus important encore, le vagabondage est entouré de mesures de précaution qui sont autant d'exceptions au droit commun. Hormis les repris de justice, il est le seul délit où la prévention interdit la libération provisoire ${ }^{22}$. De plus, après leur peine, les détenus restent à la disposition du gouvernement pour une durée indéterminée, à l'appréciation de l'administration ${ }^{23}$. Or, cette mise à disposition, souvent assortie de mesures contraignantes (prolongation de détention, interdiction de certains lieux, etc.), était conçue comme un élément central de la pénalité. La Cour de cassation le rappelle dès $1823^{24}$, F. Hélie et A. Chauveau le confirment dans leur traitée 25 . Elle survit d'ailleurs à la réforme pénale de 1832, sous la forme modifiée de la surveillance de la haute police ${ }^{2726}$. Enfin, l'article 272 prévoit que les étrangers condamnés pour vagabondage peuvent être expulsés du territoire. 
Pour justifier pareilles mesures, les juristes soulignent certes le refus coupable de se soumettre aux lois sociales, mais ils insistent aussi sur le caractère préventif du délit. Comme l'association de malfaiteurs, c'est au titre des « faits préparatoires » ou des « actes préparatoires d'autres crimes et délits » que le vagabondage doit être sanctionné ${ }^{27}$. Sans revenir sur l'étiologie du crime qui s'impose en ce début de XIX ${ }^{e}$ siècle, rappelons que c'est dans le désordre des existences populaires qu'il est censé prendre corps ${ }^{28}$. Or, le vagabondage, en ce qu'il atteste du goût d'une vie déréglée sinon aventureuse, au mépris des obligations sociales, est le symbole de ces inclinations qui portent le peuple au crime. Il est aussi le lieu de toutes les collusions possibles entre catégories populaires et ennemis de l'ordre légal, détenus libérés, malfaiteurs en fuite... F. Hélie et A. Chauveau l'expriment ainsi :

«Comment [les vagabonds] n'inspireraient-ils pas de crainte quand leur position et leurs besoins leur font en quelque sorte une loi impérieuse du crime et que leurs courses incessantes, en effaçant les traces de leurs pas, les dérobent aux recherches de la justice? $»^{29}$

9 En incriminant un état d'incertitude sociale, le vagabondage fonctionne donc comme une arme policière redoutable permettant de saisir, d'interroger, éventuellement de sanctionner tous les individus regardés comme autant de malfaiteurs potentiels du fait de leur mode de vie. Il fut ainsi mobilisé contre des populations aux ressources fragiles, jugées trop incertaines, mal assurées. À l'image du jeune Béasse, qui se targuait d'avoir 36 états $^{30}$, le vagabondage fut opposé à de nombreux individus vivant de petits métiers, en ville ou sur les routes, souvent à la frontière de la misère et de l'illégalité : marchands ambulants et petits travailleurs itinérants sont des profils qui reviennent fréquemment ${ }^{31}$ et, sous la Restauration, les colporteurs furent explicitement recommandés à l'attention des parquets ${ }^{32}$. Le délit fut aussi convoqué contre divers groupes vivant de ressources illicites, inavouables: prostituées clandestines ${ }^{33}$, mendiants étrangers aux localités ${ }^{34}$, bonneteurs et petits escrocs ${ }^{35}$. Il fut, enfin, particulièrement utile dans la traque de certains malfaiteurs, voire de déserteurs ou d'« intrigants » politiques. En 1826, J. Dufour est détenu comme vagabond car on le soupçonne d'être un voleur ou un criminel en fuite ${ }^{36}$. En 1822, P. Bailly et I. Rossfelder sont, eux, interpellés et retenus car on suppose qu'ils peuvent être des retardataires ou des déserteurs ${ }^{37}$. Délit à spectre large, le vagabondage permit donc d'atteindre bien plus que le vagabondage, en fait tous les individus suspects d'affinités avec le désordre et le crime, unis dans une même incapacité à répondre de leur mode d'existence.

\section{Au service d'une police des circulations et des populations pauvres : le contrepoint d'un important appareil réglementaire}

10 Au-delà de ces spécificités juridiques, le vagabondage participe d'un dispositif plus général d'encadrement des mobilités. Sans revenir sur l'histoire de l'identification ou de l'assistance, il est nécessaire de souligner à quel point le délit, dans ses modalités d'application, est tributaire du cadre tracé par la réglementation.

\section{Passeports et papiers}

11 Dans la lignée de l'Ancien Régime, les papiers sont toujours au cœur du dispositif de contrôle des mobilités: imposé à tout voyageur, le passeport intérieur doit garantir identification et suivi des déplacements, tout en assurant le partage entre les circulations 
licites, validées, et celles, illicites, qui entraînent une présomption légale de vagabondage ${ }^{38}$. Aussi l'arrestation des vagabonds résulte généralement de leur incapacité à produire des papiers en règle et, dès qu'il s'agit de renforcer la répression, les passeports sont la première arme mobilisée. En 1817, dans un contexte marqué par les difficultés frumentaires, alors qu'il s'agit de contenir les manifestations de la pauvreté, d'éviter qu'elles n'entraînent des circulations incontrôlées, ministres et préfets rappellent la nécessité de tenir la main à la vérification des papiers et à l'arrestation des vagabonds ${ }^{39}$. Vagabondage et passeports sont donc deux instruments d'une même police que les autorités convoquent à des degrés variables, suivant les circonstances. Dès que les circulations deviennent massives, inquiétantes, ou que le contexte politique le réclame, on invite les maires à restreindre la délivrance des passeports, et la menace d'une arrestation pour vagabondage est agitée ${ }^{40}$. C'est le cas en 1822, lors de la foire de Beaucaire $^{41}$. Cela l'est encore en 1831, lorsque, à la suite de vols et d'incendies, plusieurs préfets renforcent les contrôles en la matière ${ }^{42}$. Les années 1848-1849 marquent cependant l'apogée de cette pratique. Afin de tenir éloignés ouvriers et individus sans travail, le gouvernement Cavaignac impose des restrictions à l'attribution des passeports en direction de Paris : nécessité d'attester d'un domicile ou d'un emploi dans la capitale, obligation d'obtenir le visa du préfet ou du sous-préfet ${ }^{43}$. Le garde des Sceaux invite ensuite les parquets à sévir, et les condamnations pour vagabondage s'envolent ${ }^{44}$. Les lois du 9 août 1849, organisant l'état de siège, et du 9 juillet 1852, sur l'interdiction de séjour, consacrent cette évolution, les autorités ayant le pouvoir d'interdire l'accès de Paris et Lyon aux repris de justice et aux individus sans ressource n'y ayant pas de domicile. Audelà des seuls passeports, les autorités cherchent aussi à renforcer la surveillance des groupes suspects par les papiers. Entre 1816 et 1824, l'obligation pour les colporteurs de se munir d'un livret est maintes fois rappelée, tout comme le risque d'une condamnation pour vagabondage en cas de contravention ${ }^{45}$. G. Haudebourg signalait, lui, le cas des marins et des militaires démobilisés ${ }^{46}$.

\section{Fixation des pauvres et partages assistantiels}

12 Le cadre d'application du délit est aussi déterminé par l'organisation assistantielle, dont un objectif majeur est, en ce début de XIX ${ }^{e}$ siècle, de fixer les pauvres dans leurs communes, à travers des secours localisés ${ }^{47}$. Cette fixation doit permettre une meilleure gestion de la pauvreté, sous le contrôle des élites locales, mais aussi de conjurer les désordres liés aux circulations, à travers l'incrimination des indigents qui persistent à s'éloigner - « mendiants étrangers » ou « mendiants nomades » que le vagabondage vient sanctionner ${ }^{48}$. La crise frumentaire de 1816-1817 est l'occasion d'affirmer cette logique duale, à valeur de paradigme: aux invitations à organiser des secours locaux, sous l'autorité de bureaux de bienfaisance, répondent les injonctions à arrêter mendiants et vagabonds saisis hors de leurs localités (commune ou canton). Dès le printemps 1816, puis durant l'hiver et au début de l'année 1817, les préfets multiplient les arrêtés et circulaires en ce sens ${ }^{49}$. Dans la Nièvre, le programme est clairement tracé :

"Ainsi donc, chaque commune aura, par la combinaison des deux mesures qui viennent d'être développées, dans ses bureaux de bienfaisance des secours assurés aux pauvres infirmes, et dans les travaux de ses chemins vicinaux [...] un moyen d'occuper chaque année ses pauvres valides, pendant la courte stagnation des travaux particuliers [Le préfet fait ici référence à la création d'ateliers de charité]. Dès lors, il ne restera plus aux indigents infirmes ou valides de prétexte pour sortir de 
leurs communes. [...]. Une fois la mendicité ainsi interdite, tout mendiant trouvé

hors de sa commune pourra être poursuivi comme vagabond $»^{50}$.

13 L'importance de cette politique est ensuite réaffirmée par le gouvernement, dès l'été 1817, entraînant une nouvelle vague d'arrêtés ${ }^{51}$. On en perçoit encore les échos sous la monarchie de Juillet. En 1831, le préfet du Tarn-et-Garonne ordonne l'expulsion des mendiants et vagabonds étrangers à son département, après avoir invité les maires à organiser des secours locaux ${ }^{52}$. Entre 1840 et 1842, les efforts déployés en Saône-et-Loire pour créer un système d'assistance dans les communes, ainsi qu'un dépôt de mendicité à Mâcon, visent d'abord à éloigner « mendiants nomades » et « vagabonds étrangers ». Les mendiants locaux peuvent, eux, bénéficier d'une certaine tolérance ${ }^{53}$. Contrepoint d'un appareil réglementaire visant à resserrer la surveillance sur les populations pauvres et les mobilités, le vagabondage devait en ressaisir les échappés.

\section{Une cible prioritaire : les individus évoluant en marge de l'ordre local}

Opposé à tous ceux dont l'existence paraît suspecte, incertaine, le vagabondage oriente le regard policier vers les inconnus de passage, étrangers au lieu. L'extranéité est la première condition d'interpellation, déclenchant la vérification des papiers. Ce point a suffisamment été éclairé par l'historiographie pour que nous y revenions ${ }^{54}$. Au-delà des agents, ces étrangers suscitent souvent l'attention des populations. En 1822, suite à un incendie, les habitants du canton de Lucenay (Saône-et-Loire) orientent les recherches vers un vagabond suspect ${ }^{55}$. De même, la tentative de vol commise à Béligny (Rhône), en 1846, est vite attribuée à un étranger que l'on avait vu rôder dans les environs ${ }^{56}$. Certaines arrestations témoignent cependant de relations plus complexes entre sociétés locales et individus de passage. Quelques-uns sont connus des habitants, qui les ont accueillis ou côtoyés, mais leur insertion demeure fragile, sujette à réévaluation en cas d'écarts ou de tensions avec la communauté. P. Bailly ou P. Garnier ont chacun vécu au contact des villageois avant qu'un incident ne provoque leur arrestation ${ }^{57}$. Celle-ci révèle alors la persistance d'une certaine défiance vis-à-vis de ces individus, maintenus dans une position d'extériorité.

Le regard policier est également orienté par une série d'archétypes. Les multiples visages de la pauvreté itinérante occupent une place de choix ${ }^{58}$ : mendiants sac au dos à l'image de C. Brenner ${ }^{59}$, vieillards esseulés, enfants vêtus de haillons tels les frères Ferrare ${ }^{60}$. D'autres types reviennent fréquemment: jeunes femmes soupçonnées de prostitution, jeunes hommes de désertion, colporteurs et petits marchands ambulants ${ }^{61}$. Souvent, les individus se signalent par un comportement suspect ou par les écarts qu'ils commettent, surpris en train de fuir ou de mendier, lors de rixes, d'escroqueries, voire de tentatives de vol $^{62}$. D'autres, enfin, provoquent la rencontre avec les autorités pour négocier secours de route et papiers, ou pour obtenir d'être incarcérés, l'hiver en particulier. En novembre 1836, X. Bouillot exige ainsi d'être arrêté sous peine de faire un mauvais coup ${ }^{63}$. Il n'est pas rare, non plus, que le vagabondage permette de saisir des "mauvais sujets ", notoirement connus, dont le passage suscite scandale ou appréhension. Aux arrestations d'individus dénoncés pour mener une vie trop licencieuse, immorale, répondent celles des bonneteurs, voleurs ou petits escrocs, signalés à l'attention des forces de l'ordre ${ }^{64}$. Repérage et saisie des suspects prennent donc appui sur un savoir policier forgé au contact des populations. Plusieurs dossiers témoignent de l'expérience acquise par les agents et les magistrats confrontés à la gestion de ces individus, qui occupe une part importante de leur temps ${ }^{65}$. 


\title{
Une police complexe et incertaine ? la gestion des vagabonds
}

\author{
Des arrestations nombreuses, des poursuites rares : un délit peu \\ judiciarisé
}

En ce premier XIX ${ }^{\mathrm{e}}$ siècle, le vagabondage est une cible majeure de l'activité policière. J. Merriman en a démontré toute l'importance dans la pratique ordinaire des agents ${ }^{66}$. Nos archives le confirment : en 1821, 48,4\% des individus détenus sans jugement ni mandat à la prison de Roanne, à Lyon, le sont pour ce motif ${ }^{67}$. À Paris, S. Delattre relevait des chiffres tout aussi importants ${ }^{68}$. Or, le délit est peu poursuivi devant les tribunaux : 2386 cas en 1825, 2738 en 1835, puis 4074 en 1845. L'augmentation est significative, mais le vagabondage ne représente que 5 à $6 \%$ des affaires jugées ${ }^{69}$. À l'inverse, les taux de renvois sont élevés, de l'ordre d'un tiers selon G. Haudebourg ${ }^{70}$. Sur les 209 dossiers que nous avons étudiés, entre 1820 et 1850, $30 \%$ seulement aboutissent à une comparution. Cet écart entre arrestations et poursuites s'explique certes par la nature « préventive » du délit : justifiées par une simple présomption, nombre d'interpellations se soldent par des relaxes. Cependant, il signifie aussi que le vagabondage relève principalement d'une gestion policière et administrative, ce que déplorent certains magistrats ${ }^{71}$. La part laissée à l'appréciation des maires et des agents, confrontés aux populations, est en effet considérable ${ }^{72}$.

\section{Accommodements, relaxes, reconductions : la gestion policière et administrative}

Or, ces derniers ont souvent tendance à privilégier diverses formes d'accommodements. Par compassion ou pour hâter l'éloignement de ces inconnus, ils leur offrent parfois secours et assistance. Sans gîte et sans emploi, N. Truquin reçoit trois sous d'un commissaire en $1843^{73}$. La Gazette des tribunaux rapporte, elle, le cas d'un jeune homme apparemment sourd et muet, qui fut placé par le maire chez un coutelier avant d'être démasqué $^{74}$. Bien des individus sont également accueillis au poste de police pour la nuit, accablés par les fatigues et la misère ${ }^{75}$, et certaines condamnations n'ont d'autre but que d'offrir à des malheureux une prise en charge minimale, pendant quelques jours ${ }^{76}$. Passeports et secours de route entrent dans le même type de transactions : en 1822, la "femme Henriette ", vagabonde libérée, obtient un passeport d'indigent sous un nom d'emprunt, bien que le maire l'ait reconnue ${ }^{77}$. C. Ray reçoit, elle, des secours de route à l'aide d'un faux récit ${ }^{78}$. Mises en garde et admonestations font également partie du registre déployé par les autorités. Interpellés en Isère en 1836, T. Baron et J. Boni sont conduits à Lyon, où le préfet suggère de les libérer après les avoir sermonnés ${ }^{79}$. De la même manière, des renseignements positifs, fussent-ils partiels, peuvent suffire à provoquer la relaxe : arrêté en 1831, G. Alvazy est relâché sur la déclaration d'un peintre qui le dit brave garçon ${ }^{80}$. À ces libérations s'ajoutent les procédures légales du cautionnement et de la réclamation. Si les cas d'enfants rendus à un parent ou remis à un tiers sont les plus nombreux ${ }^{81}$, elles concernent aussi certains adultes. A. Malamonette, interpellé dans le Rhône en 1844, est libéré à la demande du maire de Grandrif (PuydeDôme), bien qu'il ait quitté la commune depuis dix ans ${ }^{82}$. La femme Bautier est, elle, relaxée en 1828 sur la requête d'une lingère qui l'a employée ${ }^{83}$. 

passeports assortis d'injonctions, ordres de reconduction ou d'expulsion, etc. Elles concernent d'abord les étrangers au royaume, régulièrement reconduits aux frontières sans avoir été jugés ou condamnés. À Lyon, la mesure est opposée aux Suisses, Savoyards et Piémontais retrouvés sans ressources dans la ville, ainsi qu'aux prostituées étrangères ${ }^{84}$ . En 1824, le ministre de l'Intérieur en recommande l'usage contre les « Mistous », bandes de vagabonds apparues dans l'Est de la France ${ }^{85}$. Alternative à une procédure coûteuse, fastidieuse, ces mesures d'expulsion permettent de contourner la nécessité d'une qualification juridique des faits. Relevant des compétences du préfet, sous la forme d'arrêtés, elles sont fréquemment sollicitées par le parquet, dès que les poursuites s'annoncent incertaines ou impossibles. En 1826, après que la chambre du conseil a prononcé le non-lieu, J.-F. Jansens est ainsi confié par le procureur au préfet, qui le fait reconduire aux frontières ${ }^{86}$. La pratique en est attestée jusqu'au début des années 1830 , avant que la Cour de cassation ne rappelle l'impératif d'une condamnation préalable ${ }^{87}$. Ces mesures de reconduction, toutefois, concernent aussi les citoyens nationaux. Devant la difficulté à établir les identités à distance ou la véracité de certains récits, les autorités privilégient la confrontation physique, directe, ordonnant de conduire tel individu devant le maire de la commune où il dit être né, ou devant le préfet du département. Arrêté en 1826 dans le HautRhin, J. Dufour est ainsi envoyé à Lyon - il dit y être né. Traduit devant le préfet puis le procureur, il est ensuite transféré à Épernay, où il est soupçonné de vol. Après vérification, il est renvoyé à Lyon et inculpé pour vagabondage ${ }^{88}$. Afin d'éviter ces démarches, les autorités préfèrent souvent délivrer des passeports avec itinéraire obligé ou injonction de se présenter à destination. En 1822, M. Auclair et M. Pinot reçoivent à Lyon un passeport avec itinéraire obligé et « feuille de route impérative " pour se rendre devant le préfet de l'Allier, où elles sont nées ${ }^{89}$. Ces procédés témoignent aussi de l'empressement des autorités à éloigner, à moindres frais, des individus susceptibles de troubler l'ordre, ou dont la gestion s'avère problématique - ainsi des prostituées ou des « idiots ", régulièrement expulsés et reconduits dans leurs communes ${ }^{90}$ . Procureurs et préfets n'hésitent pas, enfin, à user des possibilités offertes par le Code pénal, de lamise à disposition à la surveillance de la haute police. En 1834, le préfet du Rhône rappelle que la condamnation de M. Vallon devait surtout permettre de l'expulser de Lyon en tant que surveillée ${ }^{91}$. En ce début de XIX ${ }^{e}$ siècle, l'ordinaire du vagabondage échappe donc encore largement à l'action des tribunaux.

\section{Comprendre l'intervention de la justice pénale}

19 Dès lors, les raisons qui décident des poursuites et des condamnations sont plus difficiles à saisir. La persistance d'une forte opacité, qu'agents et magistrats n'arrivent pas à percer, revient souvent. Soupçonnant une volonté de dissimulation coupable, les tribunaux prononcent des peines lourdes, généralement assorties d'une mise sous surveillance. P. Trouillé, C. Mercier ou X. Bouillot sont condamnés à plusieurs mois de prison et à cinq ans de surveillance pour n'avoir pu fournir des renseignements valables ${ }^{92}$. Quelques années plus tôt, en 1828, la «femme sans nom ", vagabonde parisienne persistant à taire son identité, était condamnée à trois mois de prison et mise à disposition du gouvernement, après cinq renvois d'audience ${ }^{93}$. Viennent ensuite les éléments signalant un "mauvais sujet ", à l'immoralité plus ou moins avérée. Le refus d'entendre avertissements ou injonctions entre dans cette catégorie. En 1836, J. Drevet est inculpé car il a ignoré le délai de quinze jours qui lui était fixé, mendiant à Villefranche- 
sur-Saône au lieu de rentrer à Saint-Étienne ${ }^{94}$. Des renseignements défavorables, attestant d'une mauvaise réputation ou d'inconduites notoires, peuvent aussi entraîner des poursuites. En 1849, M. Tory est condamné à quinze jours de prison pour vagabondage et mendicité habituelle, le commissaire ayant souligné sa paresse et sa tendance à courir la campagne ${ }^{95}$. Enfin, plusieurs individus sont inculpés pour avoir persisté dans des comportements répréhensibles, qui leur avaient déjà valu d'être sanctionnés. J.-B. Monsu, à peine sorti de prison pour vagabondage, est à nouveau arrêté en janvier 1831 avec d'autres mendiants. Il est condamné à six mois de prison ${ }^{96}$. L'existence d'antécédents est certes déterminante mais, en l'absence de casier judiciaire, elle dépend étroitement de la mémoire des agents et de la sagacité des magistrats. J. Trouvé, qui cachait son nom, est ainsi reconnu par le procureur qui l'a déjà fait condamner. Il lui en coûte treize mois de prison $^{97}$. Des faits aggravés ou des atteintes trop manifestes à l'ordre public, quand il s'agit d'individus étrangers, à la situation incertaine, peuvent aussi entraîner des poursuites pour vagabondage. C'est le cas de la mendicité en réunion ou avec me- naces, des escroqueries et des rixes, des tentatives de vol, voire de la prostitution clandestine. Le vagabondage se substitue alors à d'autres chefs d'accusation, plus difficiles à établir, ou s'y accole. A. Troubadier, surpris de nuit dans la cour d'une maison, est inculpé de tentative de vol, mendicité et vagabondage. Il est finalement condamné pour ce dernier chef à six mois de prison et cinq ans de surveillances ${ }^{98}$. Délit à spectre large, le vagabondage relève donc de formes de gestion variées, à la croisée de différents appareils, ce qui suppose des efforts de coordination qui ne vont pas sans heurts.

\section{L'action répressive, entre collaboration et divergences}

20 La nécessité de cette collaboration se lit dans les échanges fréquents qu'entretiennent forces de police, magistrats et autorités administratives. Au-delà des cas individuels, en particulier des étrangers, objets d'une importante correspondance ${ }^{99}$, il faut aussi mentionner les nombreuses lettres et circulaires visant à mobiliser les agents. Ministres de l'Intérieur et préfets usent d'efforts considérables pour expliciter la réglementation et convaincre les maires de l'importance de cette police des circulations ${ }^{100}$. Ils interviennent aussi, au besoin, pour faciliter l'action des magistrats. En décembre 1848, à la demande du parquet, le préfet de police de Paris organise un service de surveillance en banlieue pour lutter contre l'afflux d'immigrants belges, qui encombrent prisons et tribunaux ${ }^{101}$. De façon symétrique, gardes des Sceaux et procureurs invitent régulièrement leurs officiers à agir de concert avec l'administration et la police. Ces efforts ne doivent pas, cependant, masquer les frictions et les heurts. Des divergences opposent fréquemment maires et préfets. En 1835, celui du Rhône reproche au maire de Condrieu d'avoir libéré trois mendiants vagabonds, provoquant une vive réponse de sa part ${ }^{102}$. Elles divisent également le corps des magistrats. Interrogé sur les relaxes prononcées dans son ressort en 1849, le procureur de Riom incrimine les juges ${ }^{103}$. Certaines sont l'écho de désaccords plus profonds, touchant à l'acception de la loi. En effet, forces de police et autorités administratives tentent régulièrement d'imposer une lecture extensive de la législation. À deux reprises, en 1822 et 1829, le préfet du Rhône prend des arrêtés qui ordonnent de poursuivre comme vagabonds diverses catégories de mendiants, passant outre la définition légale des délits ${ }^{104}$. De même, lors de certains épisodes répressifs, les forces de police tentent de s'affranchir des limites fixées par le Code pénal. Tandis qu'ils multiplient les arrestations d'individus interpellés sans papiers réguliers, les agents exigent en même temps, afin de défendre leur action et de préserver leur autorité, que 
ces arrestations débouchent sur des condamnations, détournant le sens de la législation (le défaut de passeport n'entraîne qu'une présomption). En 1849, le préfet de l'Allier demande explicitement au ministre de l'Intérieur d'agir en ce sens, requête aussitôt transmise au garde des Sceaux ${ }^{105}$. Les relaxes sont, elles, très mal vécues ${ }^{106}$. Confrontés à ces pressions, les magistrats adoptent des réponses ambivalentes. Le parquet entend bien sévir pour appuyer l'action dissuasive de la police. Aux bandes de vagabonds parcourant son ressort en 1829, le procureur de Mortagne (Orne) oppose son intention de les poursuivre avec vigueur ${ }^{107}$. De même, en novembre 1848, le parquet de Paris multiplie les poursuites à l'encontre des migrants belges, afin de faciliter leur expulsion ${ }^{108}$. Les magistrats, toutefois, font aussi valoir les contraintes propres à l'exercice de leur métier. En plus des difficultés matérielles (prisons et tribunaux surchargés, effectifs insuffisants), ils insistent sur les règles de la pratique et de la procédure juridiques, à commencer par la nécessité de qualifier précisément les faits. Invité à justifier certaines relaxes, le procureur de Fontainebleau rappelle que la présomption à l'origine de l'arrestation ne résiste pas toujours à l'instruction, conduisant à des remises en liberté parfaitement fondées ${ }^{109}$.

\section{Une gestion circonstanciée ? Limites et aléas de la lutte contre le vagabondage}

21 Au-delà de ces tensions, la répression du vagabondage obéit aussi à des rythmes et à une géographie qu'il nous faut essayer d'apprécier.

\section{Espaces, temporalités : une police d'intensité inégale}

Éclairée par les statistiques officielles (CGAJC), la géographie du vagabondage reflète un double ordre de réalité, inextricablement mêlé : d'un côté, la prépondérance de certaines régions urbaines ou à forte activité industrielle, manufacturière, abritant une population de migrants et d'ouvriers aux revenus et au statut incertains; de l'autre, les efforts déployés par les autorités pour conjurer les inquiétudes liées à la présence de ce prolétariat, en usant de la répression pénale. Derrière le ressort de Paris dont la prépondérance est écrasante, seules les cours d'appel de Lyon et Colmar, situées dans des zones frontalières marquées par une forte activité industrielle (textile), enregistrent des taux significatifs ${ }^{110}$. Rennes fait cependant exception jusqu'en 1835 , sans doute en raison de la présence endémique de mendiants, voire de marins ou de militaires démobilisés, et de l'étendue du ressort ${ }^{111}$. À une autre échelle, les arrestations éclairent la carte de la vigilance policière: centres-villes et chefs-lieux, grands axes de circulations ${ }^{112}$. On retrouve aussi les espaces associés à la surveillance des déplacements: cabarets et auberges, portes des villes, foires et marchés ${ }^{113}$. Dans les grands centres urbains, les interpellations disent l'intensité d'une vie d'extérieur que la police tente de contenir: individus arrêtés sur les places et les quais, dans les chantiers ou les commerces, etc. Hors de ces lieux, la surveillance continue de relever des populations, qui interviennent parfois directement ${ }^{114}$.

La lutte contre le vagabondage obéit de même à certaines temporalités. Aux moments de moindre activité répressive répondent des phases d'accélération. Les périodes d'inquiétude et de fragilité politiques en sont une, à l'image des débuts de la Restauration. Marquées par les difficultés frumentaires, les années 1816-1817 le sont aussi par les peurs 
politiques héritées des épisodes révolutionnaire et impérial ${ }^{115}$. Elles se traduisent par des appels répétés à combattre la malveillance et ses agents, souvent assimilés aux vagabonds et aux étrangers parcourant les campagnes ${ }^{116}$. Police des circulations et répression du vagabondage revêtent alors une importance cruciale - Decazes le rappelle en août $1817^{117}$. Ministres et préfets ordonnent de renforcer la surveillance des groupes mobiles et des populations suspectes: militaires circulant sans titres, colporteurs sans livret, groupes d'étrangers vite assimilés à des mendiants, dont on réclame l'expulsion ${ }^{118}$. Afin de lutter contre les mendiants vagabonds, les autorités tentent également d'organiser l'assistance, et les parquets veillent à la répression des pauvres qui persistent à circuler ${ }^{119}$. Les années 1848-1849 offrent un autre exemple d'accélération répressive liée à des inquiétudes politiques. Révolution de Février et journées de Juin ont réveillé la crainte des circulations populaires et des agglomérations d'ouvriers. Autorités administratives et parquets agissent alors de concert pour endiguer le flux de migrants vers Paris, ce dont témoigne la hausse des poursuites et des expulsions ${ }^{120}$. La lutte contre le vagabondage peut aussi être réactivée par l'arrivée d'un nouveau personnel politique, suivant un certain volontarisme. C'est le cas pour le Rhône, en 1822, avec l'accession du comte de Tournon à la préfecture : plusieurs circulaires rappellent aux maires leurs obligations en matière de police des circulations et, dès février, un arrêté interdit la mendicité dans le département ${ }^{121}$. Devant la crise des ateliers de soie, le préfet organise des patrouilles afin de chasser les vagabonds ${ }^{122}$. Les arrestations sont nombreuses, comme les ordres de reconduction et d'expulsion ${ }^{123}$. Les périodes de difficultés économiques et de crises frumentaires sont d'autres temps forts de la répression. En 1816-1817 comme en 1829-1830, manque de travail et chertés provoquent l'apparition de bandes de mendiants et de vagabonds inquiétant les populations ${ }^{124}$. Les autorités répondent alors par une mobilisation accrue des appareils policier et judiciaire. C'est le cas dans le ressort de Lyon au printemps 1817, ou dans celui de Mortagne, en 1829125. Enfin, la lutte contre le vagabondage connaît aussi, ponctuellement, des accélérations lors de crises sécuritaires. En 1834, une série d'« attentats » commis sur les routes et dans la ville de Lyon pousse le préfet à organiser des perquisitions en banlieue, afin d'arrêter et d'éloigner les vagabonds 126.

24 Instrument d'une police qui se veut préventive des désordres, le vagabondage fonctionne donc à des niveaux d'intensité variable. Son usage dépend également de certaines limites, dans le cadre d'une société encore peu étatisée.

\section{Insuffisances et limites : une étatisation encore incertaine?}

Parmi ces limites, les insuffisances de l'appareil répressif sont souvent mises en avant, de la faiblesse des effectifs au manque de formation de certains agents ${ }^{127}$. Elles conduisent régulièrement les autorités à s'avouer désarmées, notamment lors des crises frumentaires ou économiques. En avril 1817, le procureur de Lyon fait état de ses difficultés à combattre les bandes de vagabonds avec aussi peu de gendarmes ${ }^{128}$. En 1829, ceux de Mortagne et de Mantes dressent le même constat ${ }^{129}$. Le faible empressement de certains agents à s'acquitter de leur mission est un autre thème récurrent, qui concerne les gardes champêtres mais aussi les maires, régulièrement accusés de privilégier les intérêts locaux au détriment de la loi. En décembre 1817, le préfet du Rhône leur reproche de compromettre son arrêté contre la mendicité en délivrant des certificats d'indigence, véritables permis de circuler ${ }^{130}$. G. Haudebourg signalait, lui, les plaintes des autorités bretonnes contre les maires visant les passeports ou accordant des secours avec trop de 
facilité131. En réponse, ces derniers font valoir les nécessités locales : faiblesse des forces de police, insuffisance des ressources de la bienfaisance, etc. On devine qu'ils cherchent également à éloigner des individus dont la présence inquiète. En 1835, le maire de Condrieu avoue qu'il a préféré faire raccompagner trois mendiants étrangers plutôt que de les inculper, sa commune ayant assez de pauvres ${ }^{132}$. Loin de s'imposer uniformément, le code et la loi doivent encore composer avec les exigences de l'ordre local.

Enfin, l'identification reste incertaine. Sur les passeports et les procès-verbaux, le signalement est très générique, peu attentif aux marques particulières, et les populations continuent de recourir à la dissimulation et aux fausses identités ${ }^{133}$.

A. Lièvre, arrêté pour vagabondage en 1825 , l'a été en 1823 sous le nom de P. Beluze ${ }^{134}$. Les papiers alimentent de même trafics et transactions. À propos des cartes de sûreté attribuées aux ouvriers des fortifications jusqu'en 1831, le maire de Lyon note qu'elles étaient de peu valeur, sans signalement ni désignation, et que les ouvriers se les échangeaient volontiers ${ }^{135}$. De leur côté, vagabonds et surveillés tentent de tromper les autorités pour obtenir passeports et secours de route. Au sujet de P. Planchon, qui dit avoir perdu ses papiers en 1836, le commissaire note qu'il «voulait sans doute s'en procurer d'autres en feignant l'ingénuité ", et qu'il les a probablement vendus ou abandonnés ${ }^{136}$. Enfin, l'identité des prévenus est souvent difficile sinon impossible à établir. En 1826, les autorités renoncent à vérifier celle d'A. Charpentier, qui n'a pu indiquer ni sa paroisse de naissance, ni l'âge ou le prénom de son père ${ }^{137}$. L'orthographe elle-même n'est pas encore fixée, ce qui ajoute à l'incertitude : J.-M. Bernard, arrêté en 1822, est libéré car on suppose qu'il s'agit de J.-M. Reynard, connu favorablement dans sa commune $^{138}$.

Au-delà de continuités manifestes avec les périodes précédentes, dans la définition du délit, dans son intrication avec l'effort d'encadrement des circulations et des populations pauvres, l'histoire du vagabondage en ce premier XIX ${ }^{e}$ siècle éclaire la complexité de cette police des existences irrégulières, incertaines, qui en constitue le cœur. À travers l'incrimination d'un état d'incertitude sociale, d'une incapacité à produire des garanties d'affiliation, le délit ouvre la voie à des usages multiples, différenciés : à l'interpellation des étrangers de passage, que leur apparence ou leur comportement rendaient suspects, répondait celle des mauvais sujets, inquiétant l'ordre local. En ville, l'arrestation prenait un tour plus routinier, jetant dans les filets de la police tous ceux qui, saisis en des lieux ou à des heures indus, appartenait à «l'infrasociété des misérables »"139. Écho de cette diversité d'usages, le délit recouvre des situations sociales tout aussi variées, qu'éclairent les archives judiciaires: journaliers en quête d'activité, petits travailleurs itinérants, malfaiteurs en fuite, vieillards ou idiots livrés à eux-mêmes, enfants en rupture avec leur famille... Si l'historiographie a souligné la pluralité des situations professionnelles et des réalités économiques rassemblées sous ce terme générique de vagabonds, en particulier au tournant des $\mathrm{XIX}^{\mathrm{e}}$ et $\mathrm{XX}^{\mathrm{e}}$ siècles ${ }^{140}$, ce travail d'appréhension de la "variété des situations sociales objectives » peut encore être prolongé, approfondi ${ }^{141}$ : l'horizon familial, les stigmates attachés à certains groupes (prostituées, repris de justice), l'importance de certaines expériences (armée, migrations) doivent également être appréciées. De façon symétrique, la complexité de cette police qui, aux prises avec une réalité sociale protéiforme, souvent opaque, est sommée d'en assumer la gestion et le tri, mérite d'être éclairée, au-delà des seules procédures pénales et de l'activité des tribunaux, dont l'importance s'affirme surtout entre la fin de la monarchie de Juillet et le début du Second Empire ${ }^{142}$. Il importe ainsi de faire toute sa place à un ensemble de 
pratiques, infra et extrajudiciaires, qui définissent les contours d'une gestion policière et administrative des vagabonds, dont la part fut longtemps essentielle ${ }^{143}$. Dans cette perspective, l'action des différents agents (forces de l'ordre, autorités administratives, magistrats), et leurs manières d'interpréter la loi à travers leur activité quotidienne, peuvent encore être précisées, dans le sillage d'études consacrées à la police ou aux procureurs ${ }^{144}$. Sans occulter la part de routine, à travers les tournées et les rondes pour les forces de l'ordre, lors d'interrogatoires ou de certaines audiences pour les magistrats (petit parquet, flagrants délits), les témoignages sont nombreux, qui attestent de l'embarras et des difficultés des agents confrontés à la gestion quotidienne de ces populations. En 1863, le procureur de Lyon voyait dans ces affaires de vagabondage l'une des tâches «les plus difficiles » du substitut présidant le petit parquet ${ }^{145}$. En fin de siècle, de nombreux magistrats réclamaient une clarification de la jurisprudence sur cette matière, jugée bien trop confuse, source d'injustice et de conflits ${ }^{146}$.

\section{BIBLIOGRAPHY}

\section{BIBLIOGRAPHIE}

About, I., Denis, V., Histoire de l'identification des personnes, Paris, La Découverte, 2010. Althammer, B., Gestrich, A. \& Gründler, J. (Eds.), The Welfare State and the "Deviant Poor" in Europe, 1870-1933, New York, Palgrave Macmillan, 2014.

Aranguiz, M., Vagabonds et errants à Montréal. Perception et prise en charge de l'errance, Montréal, Presses du RCHTQ, 2000.

Aubin, G. \& Gallinato, B. (Dirs.), Les espaces locaux de la protection sociale : études offertes au professeur Pierre Guillaume, Paris, Association pour l'histoire de la Sécurité sociale, 2004.

Avon-Soletti, M.-T. (Dir.), Des vagabonds aux S.D.F. Approches d'une marginalité, Saint- Étienne, Publications de l'Université de Saint-Étienne, 2002.

Beier, A.-L., Masterless Men : The Vagrancy Problem in England (1560-1640), Londres, Methuen, 1985.

Beier, A. L. \& Ocobock, P. (Eds.), Cast Out: Vagrancy and Homlessness in Global and Historical Perspective, Athens, Ohio University Press, 2008.

Berlière, J.-M. \& Lévy, R., Histoire des polices en France de l'Ancien Régime à nos jours, Paris, Nouveau Monde, 2013.

Black, J. E., A Crime to Live Without Work : Free Labor and Marginal Workers in Industrial Chicago, 1870 to 1920, Michigan Historical Review, vol. 36, n² 2, automne 2010, p. 63-93.

Blanc-Chaléard, M.-C., Douki, C., Dyonet N. \& Milliot, V. (Dirs.), Police et migrants. France, 1667-1939, Rennes, Presses universitaires de Rennes, 2001.

Bourguignat, N., « La ville, la haute police et la peur : Lyon entre le complot des subsistances et les manœuvres politiques en 1816-1817 », Histoire urbaine, 2000, n 2, p. 131-147.

Bourguignat, N., Les grains du désordre. L'État face aux violences frumentaires dans la première moitié du XIX' siècle, Paris, EHESS, 2002. 
Brodiez-Dolino, A., Combattre la pauvreté. Vulnérabilités sociales et sanitaires de 1880 à nos jours, Paris, CNRS Éditions, 2013.

Buchner, T., Mejstrik, A. \& Wadauer, S. (Eds.), The History of Labour Intermediation. Institutions and Finding Employment in the Nineteenth and Early Twentieth Centuries, New York, Oxford, Berghahn, 2015.

Castel, R., Les métamorphoses de la question sociale. Une chronique du salariat, Paris, Gallimard, 1995.

Chevalier, L., Classes laborieuses et classes dangereuses à Paris pendant la première moitié du XIX ${ }^{e}$ siècle, Paris, Hachette, 1984.

Davis, J., « Urban Policing and its Objects : Comparative Themes in England and France in the Second Half of the Nineteenth Century ", in Emsley, C., Weinberger, B. (Eds.), Policing Western Europe. Politics, Professionalism and Public Order, 1850-1940, New York/Wesport/Londres, Greenwood Press, 1991, p. 1-17.

De Koster, M. \& Reinke, H., Policing Minorities, in Johansen, A., Knepper, P. (Eds.), The Oxford Handbook of the History of Crime and Criminal Justice, New York, Oxford University Press, 2016, p. $268-284$.

Delattre, S., Les douze heures noires. La nuit à Paris au XIX siècle, Paris, Albin Michel, 2000.

Denis, V., Une Histoire de l'identité. France, 1715-1815, Seyssel, Champ Vallon, 2008. Denis, V. \& Milliot, V., « Police et identification dans la France des Lumières », in Vos papiers! Genèses, $\mathrm{n}^{\circ} 54$, 2004/1, p. 4-27.

Depastino, T., Citizen Hobo. How a Century of Homelessness Shaped America, Chicago, Chicago University Press, 2005.

Depauw, J., « Pauvres, pauvres mendiants, mendiants valides ou vagabonds ? Les hésitations de la législation royale ", Revue d'Histoire Moderne et Contemporaine, juillet-septembre 1974, p. 401-418.

Derasse, N., Dubois, B., Durand, B., Jean, J.-P. \& Royer, J.-P., Histoire de la justice en France : du XVIII ${ }^{e}$ siècle à nos jours, Paris, Presses universitaires de France, 2010.

Emsley, C., Crime, Police and Penal Policy. European Experiences (1750-1940), Oxford/ New York/ Auckland, Oxford University Press, 2007.

Farcy, J.-C., « Le procureur entre l'ordre public et les justiciables : plaintes, procès-verbaux et poursuites pénales à Dijon à la fin du XIX siècle », Crime, Histoire et Société, vol. 9, n 1, 2005, p. 79-115.

Foucault, M., Surveiller et punir. Naissance de la prison, Paris, Gallimard, 1975.

Gaboriau, P., S.D.F. à la Belle époque. L'univers des mendiants vagabonds au tournant des XIX ${ }^{e}$ et XX ${ }^{e}$ siècles, Paris, Desclée de Brouwer, 1998.

Gaume, P., Ces pauvres menaçants : vagabondage et pénalisation des classes populaires au XIXe siècle (années 1820-1912), mémoire de Master II (sciences sociales, EHESS), 2009.

Geremek, B., La potence ou la pitié. L'Europe et les pauvres du Moyen Âge à nos jours, Paris, Gallimard, 1987.

Golliard, O., « Dépénaliser le vagabondage ? L'impact relatif du décret-loi d'octobre 1935 », Criminocorpus, mis en ligne le 2 septembre 2014. [URL : http://criminocorpus.revues. org/2761] Gueslin, A., Gens pauvres, pauvres gens dans la France du XIX ${ }^{e}$ siècle, Paris, Aubier, 1998.

Gueslin, A., D'ailleurs et de nulle part : mendiants, vagabonds, clochards, SDF en France depuis le Moyen Âge. Paris, Fayard, 2013. 
Gutton, J.-P., La société et les pauvres en Europe (XVI ${ }^{e}-\mathrm{XVIII}{ }^{e}$ siècles), Paris, Presses universitaires de France, 1974.

Haudebourg, G., Mendiants et vagabonds en Bretagne au XIX ${ }^{e}$ siècle, Rennes, Presses universitaires de Rennes, 1998.

Higbie, F. T., Indispensable Outcasts : Hobo Workers and Community in the American Midwest, 1880-1930, Urbana/Chicago, University of Illinois Press, 2003.

Jung, B., La bataille du placement et les sans-travail. Concurrences entre intermédiaires et synthèse républicaine dans la genèse du marché du travail en France (1880-1914), thèse de doctorat (Histoire, Paris VII), 2012.

Kalifa, D., Crime et culture au XIX ${ }^{e}$ siècle, Paris, Perrin, 2005. Kalifa, D., Les basfonds. Histoire d'un imaginaire, Paris, Seuil, 2013.

Kusmer, K. L., Down and Out, on the Road : The Homeless in American History, New York, Oxford University Press, 2002.

Lawrence, P., « Policing the Poor in England and France, 1850-1900 ", in Emsley, C., Johnson, E. \& Spierenburg, P. (Eds.), Social Control in Europe, volume $2: 1800-2000$, Athens, Ohio University Press, 2004, p. 210-225.

Lawrence, P., " The Police and Vagrants in France and England during the Nineteenth Century ", in Livio Antonelli (Dir.), Polizia, ordine pubblico e crimine tra città e campagna : un confronto comparativo, Soveria Mannelli, Rubbettino, 2010, p. 49-60.

Lawrence, P., «The Vagrancy Act (1824) and the Persistence of Pre-emptive Policing in England since $1750 »$, British Journal of Criminology, 2016, 19 p.

Lévy, R., « Un aspect de la mutation de l'économie répressive au XIX ${ }^{\mathrm{e}}$ siècle : la loi de 1863 sur le flagrant délit », Revue historique, tome 274, fasc. 1 (555), juillet-septembre 1985, p. 43-77.

Mansfield, M., Salais, R. \& Whiteside, N. (Dirs.), Aux sources du chômage. 1880-1914, Paris, Belin, 1994.

Marec, Y., Pauvreté et protection sociale aux XIX $X^{e}$ et XX $X^{e}$ siècles. Des expériences rouennaises aux politiques nationales, Rennes, Presses universitaires de Rennes, 2006.

Marec, Y. \& Petit, J.-G. (Dirs.), Le social dans la ville en France et en Europe (1750-1914), Paris, Éditions de l'Atelier, 1996.

Merrien, F.-X., Face à la pauvreté. L'Occident et les pauvres hier et aujourd'hui, Éditions de l'Atelier, 1994.

Merriman, J., Polices Stories. Building the French State, 1815-1851, New York, Oxford University Press, 2006.

Milliot, V., « Réformer les polices urbaines au siècle des Lumières : le révélateur de la mobilité », Crime, Histoire et Société, vol. 10, nº 1, 2006, p. 25-50.

Monkkonen, E. H., Walking to Work: Tramps in America, 1790-1935, University of Nebraska Press, 1984.

Noiriel, G. (Dir.), L'identification. Genèse d'un travail d'État, Paris, Belin, 2007. Ringenbach, P., Tramps and Reformers, 1873-1916: The Discovery of Unemployment in

NewYork, Westport, Greenwood Press, 1973.

Roche, D. (Dir.), La ville promise. Mobilité et accueil à Paris (fin XVII ${ }^{e}$-début XIX ${ }^{e}$ siècle), Paris, Fayard, 2000. 
Roche, D., Humeurs vagabondes. De la circulation des hommes et de l'utilité des voyages, Paris, Fayard, 2003.

Roman, D., Le droit public face à la pauvreté, Paris, L.G.D.J, 2002.

Rose, L., Rogues and Vagabonds. Vagrant Underworld in Britain (1815-1985), Londres/New York, Routledge, 1988.

Saillard, A., «L'Autre dans les mécanismes étatiques de contrôle de la mobilité. (France, seconde moitié du XIX ${ }^{\mathrm{e}}$ siècle) », Politique européenne, 2015/1, n 47, p. 94-120.

Schnapper, B., « La répression du vagabondage et sa signification historique du XIV ${ }^{\mathrm{e}}$ au XVII ${ }^{\mathrm{e}}$ siècle », Revue de droit français et étranger, tome 63, 1985, p. 143-157.

Smith, T. B., « Assistance and Repression : Rural Exodus, Vagabondage and Social Crisis in France, 1880-1914 ", Journal of Social History, vol. 32, n 4, été 1999, p. 821-846.

Smith, T. B., " The Plight of the Able-Bodied Poor and the Unemployed in Urban France, 1880-1914 », European History Quarterly, vol. 30 (2), avril 2000, p. 147-184.

Thuillier, G., Préfets et mendiants : le dépôt de mendicité de la Nièvre (1808-1820), Paris, Comité d'Histoire de la Sécurité sociale, 2002.

Topalov, C., Naissance du chômeur, 1880-1910, Paris, Albin Michel, 1994.

Wadauer, S., « Establishing Distinctions : Unemployment versus Vagrancy in Austria from the Late Nineteenth Century to 1938 », International Review of Social History, vol. 56 (1), avril 2011, p. 31-70.

Wagniart, J.-F., « Les migrations des pauvres en France à la fin du XIX ${ }^{\mathrm{e}}$ siècle : le vagabon- dage, ou la solitude des voyages incertains ", Genèses, 1998, vol. 30 (1), p. 30-52.

Wagniart, J.-F., Le vagabond à la fin du XIX siècle, Paris, Belin, 1999.

Wagniart, J.-F., « La gendarmerie et les gendarmes face à la question du vagabondage (1871- 1914) ", in Jean-Noël Luc (Dir.), Gendarmerie, État et société au XIX siècle, Paris, Publications de la Sorbonne, 2002, p. 289-299.

Yvorel, J.-J., « Vagabondage des mineurs et politique pénale en France de la Restauration à la République des ducs ", in Caron, J.-C., Stora-Lamarre, A. \& Yvorel, J.-J. (Dirs.), Les âmes mal nées. Jeunesse et délinquance urbaine en France et en Europe (XIX ${ }^{e}-X X^{e}$ siècles), Besançon, Presses universitaires de Franche-Comté, 2008, p. 63-83.

\section{NOTES}

1. Archives départementales du Rhône (AD 69), 3K 09, circulaire du préfet du Rhône (circ. P69), 20 février 1821.

2. Le terme d'«étrangers " renvoie ici aux individus extérieurs à la communauté locale, «horsains " ou «forains " évoluant hors de la sphère habituelle d'interconnaissance des populations.

3. Outre Castel (1995) ; Geremek (1987); Gutton (1974), citons : Depauw (1974); Gueslin (2013); Schnapper (1985). Pour les études récentes consacrées à la question des mobilités et des procédures de surveillance, cf. Blanc-Chaléard, Douki, Dyonet, Milliot (Eds.) (2001) ; Denis (2008) ; Denis, Milliot (2004) ; Milliot (2006) ; Noiriel (Dir.) (2007, p. 79-96); Roche (Dir.) (2000) ; Roche (2003). 
4. Davis (1991) ; De Koster \& Reinke (2016) ; Lawrence (2004); Merriman (2006). Concernant la maréchaussée et la gendarmerie, $c f$. Dyonet, N. et Houte, A., in Blanc-Chaléard, Douki, Dyonet, Milliot (Eds.) (2001, p. 51-62, 235-249) ; Wagniart (2002).

5. Sur cette caractéristique du délit de vagabondage, mise en évidence par des juristes américains dès les années 1950, cf. Beier (1985) ; Beier, Ocobock (Eds.) (2008) ; Chassaing, J.-P., in Avon-Soletti (Dir.) (2002, p. 15-21).

6. Ibidem ; Derasse, Dubois, Durand, Jean \& Royer (2010, p. 554-556) ; Lawrence (2016).

7. Pour le début du XIX ${ }^{\mathrm{e}}$ siècle, la Gazette des tribunaux (GDT) offre de nombreux exemples de ces audiences «à la chaîne ": cf. GDT, $\mathrm{N}^{\circ} 122,19$ mars 1826, $\mathrm{N}^{\circ} 454,7$ mars 1827, $\mathrm{N}^{\circ} 1284,20$ sept. 1829.

8. Lévy (1985).

9. Althammer, B., in Althammer, Gestrich \& Gründler (Eds.) (2014, p. 103-125) ; Gaboriau (1998) ; Gueslin (2013) ; Smith (1999, 2000); Wagniart (1999). Voir également, dans une perspective compa- ratiste concernant cette période: Aranguiz (2000); Black (2010); Depastino (2005) ; Higbie (2003) ; Kusmer (2002) ; Mansfield, Salais \& Whiteside (Eds.) (1994, p. 41-62) ; Monkkonen (1984) ; Rin- genbach (1973) ; Rose (1988) ; Wadauer (2011) ; Wadauer, S., in Buchner, Mejstrik \& Wadauer (Eds.) (2015, p. 286-334).

10. Buchner, Mejstrik \& Wadauer (Eds.) (2015) ; Jung (2012) ; Mansfield, Salais \& Whiteside (Eds.) (1994) ; Smith (1999, 2000) ; Topalov (1994); Wagniart (1999).

11. Les dossiers du tribunal de Lyon, non conservés, n'ont pu être consultés.

12. Gaume (2009).

13. La première expression est de Morin, A., Répertoire général et raisonné du droit criminel, Paris, Durand, 1851, tome 2, p. 393. Pour la seconde, cf. Golliard (2014).

14. Le caractère essentiellement « conventionnel » du délit de vagabondage, visant un genre de vie plus qu'un fait réellement délictueux, est signalé par la plupart des juristes du XIX ${ }^{\mathrm{e}}$ siècle. $C f$. Bonnin, P., Commentaire du Code pénal et des lois de la presse, Paris, Cotillon, 1845, p. 228-237; Chauveau, A., Hélie, F., Théorie du Code pénal, Paris, Cosse, 1852, tome 3, p. 267-317 ; Bonne, H., La répression du vagabondage et de la mendicité, Paris, Millot frères et Cie, 1900. Concernant l'historiographie, cf. Beier, Ocobock (2008, p. 134) ; Chassaing, J.-P., in Avon-Soletti (Dir.) (2002, p. 15-21).

15. Ibid., p. 19. Selon l'art. 270 sont reconnus « vagabonds ou gens sans aveu » « ceux qui n'ont ni domi- cile certain, ni moyen de subsistance, et qui n'exercent habituellement ni métier ni profession ».

16. AD 69, 4M 189 (1826), dossier M. Raymond, lettre du directeur général de la police (DGP), 11 fé- vrier 1826.

17. Id., tribunal correctionnel de Villefranche-sur-Saône (TCV), UV 1208 (1832), dossier de procédure $(\mathrm{dP})$ de $\mathrm{P}$. Trouillé.

18. Id., 4M 190 (1831), dossier F. Massot ; id., 4M 185 (1822), dossiers M. Auclair, J. Vercker.

19. Valentin-Smith, J.-E., De la mendicité et du travail, Clermont, Thibaud-Landriot frères, 1848, p. 7677.

20. Dès 1835, elles sont prononcées dans plus de 25 pour cent des cas. En 1845, la proportion dépasse $60 \%$. Voir le Compte général de l'administration de la justice criminelle en France (CGAJC) pour ces deux années.

21. Art. 277 du Code pénal.

22. Art. 115 du Code d'instruction criminelle.

23. Art. 271 du Code pénal.

24. Cf. Carnot, M., Commentaire sur le Code pénal, Paris, Nêve, 1836, p. 732.

25. Chauveau, A., Hélie, F., op. cit., tome 3, p. 279.

26. Duvergier, J.-B., Code pénal annoté. Édition de 1832 [...], Paris, Guyot et Scribe, 1833, p. 45-46.

27. Bonnin, P., op. cit., p. 228 ; Chauveau, A., Hélie, F., op. cit., tome 3, p. 254. 
28. Chevalier (1984); Emsley (2007); Kalifa (2005, 2013); Lawrence (2010).

29. Chauveau, A., Hélie, F., op. cit., tome 3, p. 268.

30. Foucault (1975, p. 296-299).

31. Sur 209 dossiers, 16 font référence à ce type d'activités.

32. CARAN, BB17a/17/11, circulaire (circ.) du ministre de la Justice (MJ), 12 juin 1822.

33. Sur 34 femmes arrêtées comme vagabondes, 15 sont accusées de se livrer à la prostitution. Voir aussi Peccoud, P., in Avon-Soletti (2002, p. 213-230) ; Merriman (2006).

34. Par exemple: AD 69, 4M 185 (1823), dossier J.-B. Cotte ; id., TCV, UV 1207 (1831), P de J. Trouvé.

35. AD 69, TCV, UV 1197 (1825), dP de C. Jarry ; id., UV 1220 (1836), dP d'A. Dupont, A. Malisson, F. Malisson, P. Malisson.

36. Id., 4M 189 (1826), dossier J. Dufour.

37. Id., 4M 185 (1822), dossiers P. Bailly, I. Rossfelder.

38. Cette présomption, liée au défaut de passeport, est prévue par la loi du 2 octobre 1795. Pour une synthèse et une bibliographie récentes sur ces questions, $c f$. About, Denis (2010).

39. CARAN, F19/940, circulaire du ministre de la Police générale (circ. min. PGle), 27 août 1817 ; circ. P69, 15 novembre 1817, in Pionin, C., Code de police municipale de la ville de Lyon, Lyon, Dumoulin, Ronet et Sibuet, 1840, p. 42.

40. Pour un parallèle avec l'Empire, $c f$. Denis, V., in Blanc-Chaléard, Douki, Dyonet, Milliot (Dirs.) (2001, p. 8283).

41. AD 69, 4M 17, circ. du ministre de l'Intérieur (MI), 20 juin 1822.

42. Le fait est attesté en Seine-Inférieure et dans le Rhône : $c f$. Marec (2006, p. 83-84) ; AD 69, 4M 191, circ. P69, 16 mars 1831.

43. Id., 3K 37, circ. P69, 9 octobre 1848, 13 novembre 1848.

44. CARAN, BB18/1467A/6314, circ. MJ, 29 novembre 1848. Le nombre de prévenus jugés passe de 6099 (1848) à 7546 (1849), puis à 8427 (1850). Cf. CGAJC 1849 et 1851.

45. AD 69, 3K6, circ. P69, 19/08/1817 ; id., 4M 17, circ. MI, 27 juin 1822.

46. Haudebourg (1998, p. 301-305).

47. Sur cette territorialisation des secours, cf. Aubin \& Gallinato (Dirs.) (2004) ; Brodiez-Dolino (2013) ; Castel (1995) ; Gueslin (1998) ; Marec \& Petit (Dirs.) (1996) ; Merrien (1994) ; Roman (2002). 48. Ces expressions sont employées de façon assez usuelle dans les textes officiels : cf. AD 69, 3K 6, circ. P69, 24 décembre 1817; AD 71, M. 7191, rapport du préfet devant le conseil général de Saône-et- Loire (CG71), session de 1842.

49. Cf. AD 69, 3K 6, circ. P69, 8 mars 1817 ; Haudebourg (1998, p. 283-284) ; Thuillier (2002, p. 267269, 292305).

50. Ibid., p. 305.

51. CARAN, F16/940, circ. MI, 28 juin /1817 ; circ. min. PGle, 27 août 1817, doc. cit. La plupart des arrêtés et circulaires sont conservés dans la série F19/940 des Archives nationales. Concernant le Rhône, cf. AD 69, 3K 6, circ. P69, 12 juillet 1817 ; circ. P69, 24 décembre 1817, doc. cit.

52. Lacoste, J.-J., Aperçu sur la question de l'extinction de la mendicité, Agen, P. Noubel, 1841, p. 18.

53. CARAN, F15/3961, rapport du préfet devant le CG71, 25 novembre 1840 ; AD 71, M. 1791, circulaire du préfet de Saône-et-Loire (P71), 3 février 1841 ; id., circ. P71, 13/10/1841; rapport du préfet devant le CG71, session de 1842, doc. cit.

54. Blanc-Chaléard, Douki, Dyonet \& Milliot (Dirs.) (2001) ; Davis (1991) ; De Koster \& Reinke (2016); Denis (2001, p. 211-332); Denis, Milliot (2004); Merriman (2006, p. 118-140); Milliot (2006); Wagniart (2002).

55. AD 71, tribunal correctionnel d'Autun (TCA), 3U 709, lettre du juge de paix de Lucenay, 05/08/1822.

56. AD 69, TCV, UV 1248 (1846), dP d'I. Rostaing.

57. Dossier P. Bailly, doc. cit. ; AD 71, TCA, 3U 710 (1823), dP de P. Garnier. 
58. Sur ce point, $c f$. Denis (2008, p. 225-242) ; Dyonet, N., et Houte, A.-D., in Blanc-Chaléard, Douki, Dyonet, Milliot (Dirs.) (2001, p. 51-62, 235-249).

59. AD 69, TCV, UV 1280 (1850), dP de C. Brenner.

60. Id., UV 1210 (1832), dP de J. et M. Ferrare.

61. Concernant la prostitution et les petits métiers ambulants : cf. supra: notes 31 à 33. Les soupçons de désertion apparaissent dans dix dossiers.

62. Sur 209 arrestations, 78 ont lieu dans ces circonstances (37,3\%).

63. AD 69, TCV, UV 1220 (1836), dP de X. Bouillot.

64. Id., UV 1191 (1822), dP d'I. Fingal, C. Mallet, J.-B. et C. Perraud ; id., UV 1197 (1825), dP de C. Demure, C. Jarry ; dP d'A. Dupont, A. Malisson, F. Malisson, P. Malisson, doc. cit.

65. Par exemple : dP de P. Trouillé, doc. cit.; AD 69, TCV, UV 1220 (1836), dP de P. Planchon.

66. Merriman (2006, p. 118-140).

67. AD 69, 4M 183 (1821), état des individus détenus dans la prison de Roanne [...]. Bien qu'élevée, la proportion est moindre dans les départements ruraux, $c f$. Haudebourg (1998, p. 306-312); Houte, A.-D., in Blanc Chaléard, Douki, Dyonet et Milliot (Eds.) (2001, p. 235-249).

68. Delattre (2000, p. 334-337).

69. CGAJC 1825,1835 et 1845 .

70. Haudebourg (1998, p. 331).

71. CARAN, BB18/972, circ. du procureur général (PG) de Rennes, 15/06/1817.

72. Davis (1991) ; De Koster, Reinke (2016) ; Lawrence $(2004,2010)$; Merriman (2006).

73. Cité par Yvorel (2008, p. 74).

74. GDT, $\mathrm{n}^{\circ} 72$ (21 janvier /1826).

75. Id., $\mathrm{n}^{\circ} 4043$ (26 août 1838).

76. C'est le cas, en particulier, des infirmes ou des vieillards: cf. GDT, n ${ }^{\circ} 938$ (09/08/1828, Dufresne), $\mathrm{n}^{\circ} 2436$ (5 juin 1833, veuve Croquelard), $\mathrm{n}^{\circ} 3797$ (10 novembre 1837, J. Mazire).

77. AD 69, 4M 185 (1822), dossier « femme Henriette ».

78. Id., dossiers L. Duval, C. Ray. Sur l'importance des passeports d'indigents et des secours de route, $c f$. Marec (2006, p. 75-86) ; Merriman (2006, p. 118-140).

79. AD 69, 4M 189 (1826), dossiers T. Baron, J. Boni, note du P69, 24 juin 1826.

80. Id., 4M 190 (1831), dossier G. Alvazy.

81. Par exemple : id., 4M 185 (1822), dossiers A. Bonnard, F. Goisque ; id., TCV, UV 1230 (1840), dPde J. Favel. Voir aussi Yvorel (2008).

82. AD 69, TCV, UV 1240 (1844), dP d'A. Malamonette.

83. $G D T, n^{\circ} 973,20$ septembre 1828 (femme Bautier).

84. Par exemple: AD 69, 4M 185 (1822), dossiers J.-B. Cotte, G. Michaud, J. Müller, A. Pierre.

85. CARAN, BB18/112/392, lettre du MI, 4/09/1824.

86. AD 69, 4M 189 (1826), dossier J.-F. Jansens.

87. Id., 4M 405, lettre du DGP, 21 juin 1833.

88. Dossier J. Dufour, doc. cit.

89. AD 69, 4M 185 (1822), dossiers M. Auclair, M. Pinot.

90. Par exemple : id., 4M 189 (1826-1827), dossier M. L'Herbette (prostituée) ; id., 4M 185 (1822), dossier J. Matthieu (« idiot »).

91. Id., 4M 192 (1834), dossier M. Vallon.

92. DP de P. Trouillé, X. Bouillot, doc. cit. ; AD 69, TCV, UV 1210 (1832), dP de C. Mercier.

93. GDT, $\mathrm{n}^{\circ} 872$ (24 mai 1828), $\mathrm{n}^{\circ} 885$ (8 juin 1828), $\mathrm{n}^{\circ} 908$ (5 juillet 1828), $\mathrm{n}^{\circ} 914$ (12 juillet 1828), $\mathrm{n}$ - 921 (19 juillet /1828), $n^{\circ} 976$ (24 septembre 1828).

94. AD 69, TCV, UV 1220 (1836), dP de J. Drevet.

95. Id., UV 1265 (1849), dP de M. Tory.

96. Id., UV 1206 (1831), dP de J.-B. Monsu. 
97. DP de J. Trouvé, doc. cit. Sur l'importance de la mémoire policière dans la reconnaissance des récidivistes, cf. Berlière, Lévy (2013, p. 139-144).

98. AD 69, TCV, UV 1220 (1836), dP d'A. Troubadier.

99. Cf. dossiers J.-B. Cotte, J.-F. Jansens, G. Michaud, J. Müller, A. Pierre, doc. cit.

100. Par exemple : circ. min. PGle, 27 août 1817, doc. cit.; AD 69, 3K 08, circ. P69, 14 novembre 1820 ; circ. P69, 20 février 1821, doc. cit.

101. CARAN, BB18/1467A/6314, lettres du MJ, 12 décembre 1848, 28 décembre 1848.

102. AD 69, 4M 193, lettre du maire de Condrieu, 17 février 1835.

103. CARAN, BB18/1467A/6314, rapport du PG Riom, 6/03/1849.

104. $A D$ 69, 3K 10, arrêté du P69, 1 février 1822 ; id., 3K 17, arrêté interdisant la mendicité, 26 janvier

1829.

105. CARAN, BB18/1467A/6314, lettre du MI au MJ, 11 février 1849.

106. Id., lettre du MI au MJ, 24 janvier 1849.

107. Id., BB18/1171/2014, lettre du procureur de Mortagne, 27 avril 1829.

108. Id., BB18/1467A/6314, rapport du PG Paris, 27 novembre 1848.

109. Id., rapport du PG Fontainebleau, 20 février 1849.

110. Paris totalise ainsi $27,7 \%$ (1830), $34,8 \%$ (1835), $41,95 \%$ (1840) et $35,8 \%$ (1845) des affaires jugées dans le pays. Concernant Colmar, les chiffres sont les suivants : 7,43\% (1830), 7,7 \% (1835), $5,66 \%(1840)$ et 7,94 \% (1845). Pour Lyon : $5,43 \%$ (1830), 4,9 \% (1835), 6,5 \% (1840) et 6,86\% (1845).

111. Rennes compte $6,8 \%$ des affaires jugées en 1830 et $7,96 \%$ en 1835 . Sa part tombe ensuite à $2,8 \%$

(1840) et $4,75 \%$ (1845).

112. Sur 110 dossiers correctionnels, 81 arrestations ont lieu sur l'axe de la Saône (Paris-Lyon).

113. 38 arrestations, soit $18,2 \%$ du total. Voir aussi Haudebourg (1998, p. 314-319).

114. AD 69, TCV, UV 1185 (1820), dP de F. Revenant; dP de C. Demure, C. Jarry, doc. cit.

115. Bourguignat (2000).

116. Ibid., p. 143 ; AD 69, 3K 5, circ. P69, 3 juin 1817.

117. Circ. min. PGle, 27 août 1817, doc. cit.

118. AD 69, 4M 17, circ. min. PGle, 9 mars 1816 ; CARAN, BB18/972/3204, circ. MJ, 25 juin 1817.

119. Cf. supra : notes 49 à 51 ; CARAN, BB18/1019/416, lettre du PG Lyon, 18 avril 1817 ; circ. PG Rennes, 15/06/1817, doc. cit.

120. Cf. supra : notes $43-44$.

121. AD 69, 3K 10, circ. P69, 31 janvier 1822 ; id., circ. P69, 8 juillet 1822 ; arrêté du P69, 1 février 1822, doc. cit.

122. AD 69, 4M 184, lettres du P69, 14 décembre 1822, 17 décembre 1822.

123. Pour la seule année 1822,40 dossiers ont été retrouvés dans les séries $4 \mathrm{M} 184$ et 185 (AD 69). Plusieurs comportent des références explicites à la politique d'expulsion des vagabonds : cf. AD 69, 4M 185 (1822), dossiers P. et E. Colombat, F. Tauny, J. Vercker.

124. Outre Bourguignat (2002), cf. CARAN, BB18/1019/416 et 495 (1817) ; id., BB18/1171/2014 et 2051 (1829).

125. Lettre du PG Lyon, 18 avril 1817, doc. cit. ; lettre du procureur de Mortagne, 27 avril 1829, doc. cit.

126. AD 69, 4M 155, lettre du P69, 9 mai 1834.

127. Cf. Berlière \& Lévy (2013).

128. Lettre du PG Lyon, 18 avril 1817, doc. cit.

129. CARAN, BB18/1171/2014, lettre du procureur de Mortagne, 4 mai 1829 ; id., BB18/1171/2051, lettre du procureur de Mantes, 26 mai 1829.

130. Circ. P69, 24 décembre 1817, doc. cit. 
131. Haudebourg (1998, p. 301-302).

132. Lettre du maire de Condrieu, 17 février 1835, doc. cit.

133. Pour une perspective historique, cf. Denis (2008, p. 383-443).

134. AD 69, TCV, UV 1197 (1825), dP d'A. Lièvre.

135. Id., 4M 209, lettre du maire de Lyon, 27 décembre 1831.

136. DP de P. Planchon, doc. cit., lettre du commissaire de police de Villefranche-sur-Saône, 15 novembre 1836

137. AD 69, 4M 189 (1826), dossier A. Charpentier.

138. Id., 4M 185 (1822), dossier J.-M. Bernard.

139. Delattre (2000, p. 337).

140. Althammer, Gestrich\& Gründler (Eds.) (2014) ; Black (2010) ; Buchner, Mejstrik \& Wadauer (Eds.) (2015) ; Jung (2012) ; Monkkonen (1984) ; Ringenbach (1973) ; Smith (1999, 2000) ; Topalov (1994) ; Wadauer (2011) ; Wagniart $(1998,1999)$.

141. Yvorel (2008, p. 75).

142. CGAJC 1880.

143. Sur l'importance de ces mesures policières et administratives dans la deuxième moitié du siècle, cf.Davis (1991) ; De Koster \& Reinke (2016) ; Lawrence $(2004,2010)$; Saillard (2015) ; Smith (1999, 2000) ; Wagniart (1999).

144. Concernant l'action des procureurs, voir par exemple : Farcy (2005).

145. AD 69, 1Y 5, lettre du procureur impérial de Lyon, 7/01/1863.

146. Exprimant un sentiment assez général, P. Pasteau, substitut du procureur à Bordeaux, écrivait en 1899: "Le délit de vagabondage sera ou non puni, ou bien plus ou moins puni, selon les sentiments plus ou moins humanitaires des magistrats [...]; plus d'harmonie serait à souhaiter et il semblerait désirable que le législateur donnât une définition plus précise ", Pasteau, P., Considérations sur les délits de vagabondage et de mendicité. [...], Bordeaux, G. Gounouilhou, 1899, p. 17.

\section{ABSTRACTS}

In nineteenth-century France, vagrancy constituted a significant aspect of public order maintenance that was written into the Penal Code of 1810. The purpose of this article is to identify the meaning and application of the law between 1815 and 1850, during the first decades when vagrancy was first included in the Penal Code. The legislation on vagrancy, which criminalised the inability to prove stable attachment to a local community and failure to register as required by law, served above all as a matrix of regulations to keep track of moving populations and the poor in order to identify those who remained unregistered. Furthermore, when vagrancy became a misdemeanour, this created great scope for policing individuals who lived in irregular and precarious conditions by intervening against a broad spectrum of behaviours and social situations. Policing the vagrant proved to be highly complex. It included an evaluation of the degree to which an individual should be regarded as suspicious as well as assessment of the general political and economic context. Policing was also shaped by the internal logic of each of the agencies (police, public authorities, and courts) who determined the individualised treatment, sometimes on the basis of the law, but very often also operating according to extra-judicial practices. 
Inscrit dans le Code pénal de 1810, le vagabondage fut un élément important du maintien de l'ordre en France au XIX ${ }^{\mathrm{e}}$ siècle. Cet article se propose d'en préciser le sens et les usages entre 1815 et 1850 , au lendemain de sa codification. Incriminant l'incapacité à répondre d'un ancrage positif dans la société et l'ordre légal, il fut d'abord le contrepoint de l'effort d'encadrement réglementaire des circulations et des populations pauvres, permettant d'en ressaisir les échappés. Au-delà, le délit ouvrait aussi la voie à une police des existences irrégulières, incertaines, recouvrant un large spectre de comportements et de situations sociales. Cette police devait s'avérer complexe: le degré de suspicion, le contexte politique ou économique, les logiques propres à chaque appareil (police, administration, justice) déterminaient des traitements différenciés, relevant encore très largement de pratiques infra et extrajudiciaires.

\section{AUTHOR}

\section{PIERRE GAUME}

Pierre Gaume est professeur agrégé d'Histoire. Doctorant à l'IRIS (EHESS), il réalise une thèse consacrée au vagabondage et à la mendicité au XIX siècle (France, années 1820-1912), sous la direction de G. Noiriel. Principale communication : « Police administrative, justice préventive ? Sens et usages du délit de vagabondage en France (1815-1850)», communication au colloque international Police et justice : le nœud gordien (1750-1850), Université de Genève, 21 novembre 2014 - pierregaume@yahoo.fr 